\title{
Cooperación y conflicto entre Argentina y Brasil: las relaciones político-diplomáticas reflejadas en los medios gráficos argentinos de 2011 a $2015^{1}$
}

\author{
Cooperation and conflict between Argentina and \\ Brazil: political-diplomatic relations reflected in the \\ Argentine graphic media from 2011 to 2015
}

\section{Ornela Fabani ${ }^{2}$, Gustavo Insaurralde ${ }^{3}$, Georgina Sabattini ${ }^{4}$}

Resumen: Tras el retorno a la democracia Brasil dejó de ser visto por la Argentina como un rival geopolítico para progresivamente transformarse en un socio deseable. Todavía más, dicha percepción mutó de manera tal que Brasil comenzó a ser juzgado como un actor indispensable para ampliar la autonomía nacional y fortalecer la inserción internacional de nuestro país. Este trabajo propone analizar las relaciones políticodiplomáticas argentino-brasileñas a la luz de sus representaciones mediáticas, prestando atención a cómo los medios gráficos argentinos han dado cuenta de las mismas en términos de cooperación o conflicto, en el período 2011-2015. En cuanto al diseño de investigación, se mixturan componentes propios de un análisis cualitativo y cuantitativo. Finalmente, las fuentes empleadas en el análisis son tanto de carácter primario como secundario.

Palabras clave: cooperación - conflicto - medios graficos -Argentina - Brasil

Abstract: After the return to democracy, Brazil stopped being seen by Argentina as a geopolitical rival to progressively become a desirable partner. Even more, this perception mutated in such a way that Brazil began to be judged as an indispensable actor to expand national autonomy and strengthen the international insertion of our country. This paper

\footnotetext{
Doi: https://doi.org/10.24215/23142766e072

${ }^{1}$ Recibido: 05/04/2019. Aceptado: 06/10/2019

${ }^{2}$ Doctora en Relaciones Internacionales por la Universidad Nacional de Rosario (UNR), Magíster en Integración y Cooperación Internacional por el Centro de Estudios en Relaciones Internacionales de Rosario (CERIR), UNR, Docente de la cátedra Política Internacional de la Facultad de Ciencia Política y Relaciones Internacionales, UNR. Correo: ornelafabanigarelli@gmail.com

${ }^{3}$ Licenciado en Relaciones Internacionales de la UNLA, cursando la Maestría en Gestión de Proyectos, Cooperación Internacional y Desarrollo para América Latina de Science Po Lyon. Actualmente, asistente de relaciones internacionales de la ENSSIB y encargado de comunicación del proyecto europeo Placed.

${ }^{4}$ Estudiante avanzada de la Licenciatura en Relaciones Internacionales en la Universidad Nacional de Rosario. Integrante del Observatorio de Política Exterior Argentina colaborando en el área de Comunicación, e integrante del Proyecto de Investigación y Desarrollo "La construcción de representaciones mediáticas de la Política Exterior Argentina a través de los medios de comunicación gráfica: el observatorio de política exterior argentina 2011-2017"
} 
proposes to analyze Argentine-Brazilian politic-diplomatic relations in the light of their media representations, paying attention to how the Argentine graphic media have reported them in terms of cooperation or conflict, in the period 2011-2015. In terms of research design, components of a qualitative and quantitative analysis are mixed. Finally, the sources used in the analysis are both primary and secondary.

Key words: cooperation - conflict - graphic media - Argentina - Brazil

\section{Introducción}

A través del tiempo podemos rastrear en la agenda de política exterior argentina distintos actores o incluso áreas geográficas con los cuales muestro país ha privilegiado sus vínculos. En este marco, cabe mencionar que, aunque las relaciones con Brasil "no siempre han figurado en el primer plano de las prioridades expresas de los gobiernos argentinos" (Jaguaribe, 1982: 9) dicho país nunca ha resultado indiferente para los tomadores de decisiones argentinos, dada su condición de nación vecina y la intensidad y variedad de los vínculos entre ambos Estados. Ahora bien, vale destacar que con el paso del tiempo ha mutado la percepción que Buenos Aires ha detentado con respecto a aquel actor. En tanto Brasilia pasó de ser visto como un rival geopolítico con el cual se competía por el control de la Cuenca del Plata y el liderazgo regional, para progresivamente transformarse en un socio deseable. Todavía más, dicha percepción mutó de manera tal que Brasil dejó de ser considerado como el principal rival geopolítico para pasar a juzgárselo como un actor indispensable para ampliar la autonomía nacional y fortalecer la inserción internacional de la Argentina (Peixoto y Loza, 2006).

En este sentido, diversos autores analizan los vaivenes y distintas etapas de las relaciones entre ambos países en distintos períodos históricos. Al respecto, Jaguaribe (1982) se remonta al germen de la relación entre estas dos naciones para concluir que el legado histórico de la época colonial y la primera mitad del siglo XX para las relaciones argentinobrasileñas fue predominantemente uno de conflicto.

Por su parte, Herrera Vegas (2012) analiza las convergencias y divergencias en las relaciones entre Argentina y Brasil desde el periodo inmediatamente posterior a la independencia hasta el gobierno de Cristina Fernández para concluir que la alianza estratégica con Brasil ha sido una de las pocas políticas de Estado de la Argentina.

No obstante, gran parte de la academia no coinciden con el análisis del autor, si tenemos en cuenta la literatura que hace hincapié en que el acercamiento entre ambos actores recién se inicia a partir de la década del $80^{\prime}$ del siglo XX, abriendo paso a una nueva fase del vínculo bilateral donde lo que predomina es la cooperación. En esta dirección, Selcher (1985) define la década del 80' como un periodo en el cual se avanza desde una cautelosa rivalidad a una competencia amistosa. En tanto, Gomez Saraiva y Tedesco (2001) analizan comparativamente las diferencias establecidas entre las líneas más generales de las políticas exteriores de Argentina y de Brasil en los años 90'. En otro orden, Bizzozero (2003) se dedica a estudiar cómo afectan los cambios de gobierno en Brasil y la Argentina en 2003 a la relación bilateral, así como también sus repercusiones sobre el MERCOSUR. Russell y Tokatlian (2005) abordan las percepciones de las elites Argentinas sobre las relaciones con Brasil desde la década del noventa en adelante, prestando particular atención a la etapa que se 
corresponde con los gobierno del Frente para la Victoria $(\mathrm{FpV})^{5}$. Este periodo también es abordado por Pereyra Doval (2014) quien define al vínculo bilateral en esta etapa como "cooperación con algunas discordias". En tanto, Actis (2015) compara la dinámica de las relaciones entre Argentina y Brasil entre 2001-2011 y 2011-2014 para sostener que la interacción bilateral atravesó, desde el año 2011, una etapa de baja intensidad relativa en términos comparativos con el periodo inmediatamente anterior.

Habiendo efectuado este recorrido no exhaustivo de la literatura relativa a la relación bilateral, lo cierto es que los especialistas coinciden respecto a que recién con el retorno a la democracia, Buenos Aires desactivó antiguas hipótesis de conflicto con los Estados vecinos, lo que le permitió un acercamiento a la región y, muy particularmente, a Brasil. Conforme con Selcher (1985), la aproximación entre estos dos actores comenzó a transitarse durante los gobiernos militares, para profundizarse a mediados de los ochentas cuando comenzó a desarrollarse cierto "nivel de mutua confianza, así como mecanismos institucionales que lograron fomentar un mayor grado de cooperación". En tanto, Bernal Meza (2008) menciona que el retorno a la democracia en Argentina surge como un elemento estructural para el cambio de las visiones sobre Brasil y las relaciones argentino-brasileñas. Estas afirmaciones cobran sentido si tenemos en cuenta que los gobiernos de Ricardo Alfonsín y José Sarney firmaron la Declaración de Iguazú (1985), que se convirtió en un punto de inflexión en la historia de las relaciones bilaterales. Años después, fruto del proceso que traccionó la firma del citado documento, se arribó a la firma del Tratado de Asunción (1991), dando origen al MERCOSUR; un organismo regional que no sólo ha apuntalado la cooperación económica sino también política entre ambos países que se reconocen como socios estratégicos. Al respecto, Peixoto y Loza (2006) señalan que la conformación del MERCOSUR generó un amplio impacto sobre el Cono Sur, en general, y estos dos países en particular, al permitir que éstos Estados se transformasen en principales socios comerciales, dando lugar a una cultura de creciente contacto e interdependencia burocrática. Todavía más, con el transcurso del tiempo los lazos con Brasil pasaron a juzgarse indispensables con vistas a un mayor grado de autonomía nacional y a una mejor inserción internacional (Simonoff, 2009; Russell y Tokatlian, 2002).

En virtud de lo expuesto se evidencia que en los últimos años, la relación con Brasil ha adquirido un lugar de relevancia primaria dentro de la agenda de política exterior argentina. En este marco, y si bien la aproximación entre ambos países lleva ya décadas, distintos especialistas han subrayado la importancia de continuar profundizando la cooperación bilateral. En esta línea, Russell y Tokatlian (2005) apuestan a la conformación de una esfera de cooperación entendida como un "mecanismo que combina elementos formales e informales para construir y mantener el orden en una región mediante la acción concertada de un núcleo de países"; siendo las principales funciones de una esfera de cooperación en una región: "proporcionar estabilidad; gestionar conflictos; conjurar crisis; difundir poder; dotar de mayor capacidad de acción a los actores menores; generar confianza; reducir incertidumbre y evitar fallas de percepción".

No obstante lo cual, también existen autores que, a pesar de respaldar esta propuesta y reconocer la importancia de desarrollar políticas de cooperación con Brasil, entienden que

\footnotetext{
${ }^{5}$ El Frente para la Victoria (FpV) es una coalición política argentina, que tiene como fuerza mayoritaria al Partido Justicialista, que fue constituida en 2003 con el objetivo de apoyar la candidatura presidencial de Néstor Kirchner
} 
el vínculo con este actor tuvo sus idas y vueltas dando lugar a que el mismo no lograse consolidarse como una "política estratégica, genuina y sostenible" (Creus, 2014: 63). Al respecto, Creus (2014) destaca que esto se ha debido fundamentalmente a las crecientes asimetrías de poder existentes en detrimento de la Argentina y a la consecuente brecha de intereses y objetivos que surgió entre las políticas exteriores de ambos Estados. Por consiguiente, podemos concluir que las relaciones bilaterales han sabido mixturar momentos así como también áreas tanto de cooperación como de conflicto.

Dicho esto, este trabajo propone analizar las relaciones político-diplomáticas argentino-brasileñas a la luz de sus representaciones mediáticas, prestando particular atención a cómo los medios gráficos argentinos han dado cuenta de las mismas en términos de cooperación o conflicto en el período 2011-2015.

Con vistas a poder desarrollar dicho estudio nos valemos de un conjunto de conceptos vitales para nuestro análisis. En primer lugar, y dado que pretendemos abordar el vínculo bilateral entre dos actores estatales, vale destacar que entendemos a la política exterior como una política pública (Ingram y Fiederlein, 1988). Dicha política se expresa en un conjunto de decisiones y acciones implementadas por los gobernantes de un Estado, en respuesta a ciertas demandas y determinantes internos tanto como externos, calculadas para cambiar o preservar las condiciones del contexto internacional siempre con el objetivo de promover los intereses y valores del Estado en el sistema internacional (Perina, 1988). Esta política pública busca otorgar visibilidad, plasmar la estrategia que se da la sociedad política, o parte de ella, para proponer modos de inserción del Estado en el contexto mundial (Miranda, 1988). Al respecto, puede afirmarse que la inserción alude a la condición de ser tomado en cuenta por la política y la economía mundial (Miranda, 2001). Cabe mencionar, que la inserción internacional ha sido una preocupación de primer orden de los gobiernos del FpV, que apostaron por alcanzarla ampliando sus márgenes de acción autónoma. Entendiéndose por autonomía "la máxima capacidad de decisión propia que puede lograr un Estado teniendo en consideración los condicionamientos objetivos del mundo real" (Puig, 1984: 42).

En esta misma dirección, lejos de la "inserción excluyente" (Miranda, 2001:172) protagonizada por la Argentina en los años 90', etapa en la que nuestro país buscó hacerse un lugar en el escenario internacional de la mano de los Estados Unidos, la estrategia de inserción internacional argentina cambió con la llegada al poder del FpV (2003-2015). Ello en virtud de que estos gobiernos percibieron la vigencia de un orden internacional "multipolar, plural, diverso y complejo" (Russell y Tokatlian, 2013: 162) y reconocieron al país como una nación en desarrollo. Tales circunstancias tuvieron como correlato que durante esa etapa se dejase de lado la búsqueda de reinserción en el denominado primer mundo, se optase por una diversificación de las relaciones externas, así como también por una política exterior de cariz autónomo en el marco de la cual Brasil ocupó un lugar de privilegio. En dicho contexto, Actis plantea que con la llegada al poder de los gobiernos de Néstor Kirchner y Lula Da Silva, en el año 2003, la relación entre Argentina y Brasil experimentó una nueva fase. Esto a partir de que tanto en la dimensión económica como política los vínculos adquirieron una fuerte intensidad favoreciendo que "ambos países ubicasen al "otro" en la centralidad de sus estrategias de inserción internacional" (Actis, 2015:28).

Por otra parte, considerando que nuestro trabajo apunta a estudiar cómo los medios gráficos han dado cuenta de la cooperación y el conflicto en la relación bilateral ArgentinaBrasil, otro concepto fundamental para nuestro análisis es el de representaciones mediáticas. Conforme con Horta (2013:97) una representación es "una expresión significativa que 
depende del contexto de producción, y cuya finalidad está depositada en la pretensión de "informar" a un alguien". Sin embargo, para el autor esto es sólo la mitad del proceso pues, para poder informar, es necesario un otro que interprete lo representado dentro de un contexto social de recepción. Como consecuencia, "la relación de elementos implicados en ambos contextos (producción-recepción) nos lleva hacia una construcción ambigua en la imagen del presente". Siguiendo a Gomis (1991: 41), la noticia es, entonces, "una representación social de la realidad cotidiana, producida institucionalmente, que se manifiesta en la construcción de un mundo posible".

En cuanto a las ideas de cooperación y conflicto que, como veremos emergen de las referidas representaciones mediáticas, se hace referencia a un conflicto cuando dos Estados pasan por un proceso de competición consciente debido a la búsqueda de valores u objetivos exclusivos. En dicho marco los competidores se transforman en rivales conscientes, oponentes o enemigos y llevan a cabo acciones con el objetivo inmediato de neutralizar, lastimar o eliminar a su contrincante (Lasswell, 1931; Williams, 1947). Tal como fue previamente referido, conforme con Jaguaribe (1982), la etapa en la cual se evidenció con mayor claridad el conflicto en el vínculo bilateral entre Argentina y Brasil fue la etapa colonial y el periodo que se extiende hasta la primera mitad del siglo XX.

Como contrapartida, la cooperación puede ser entendida como las acciones que reflejan ajustes de comportamiento a las preferencias reales o anticipadas de otros a través de un proceso de coordinación política (Keohane, 1986; Milner, 1992; Battistela, 2015).

En lo que atañe al diseño de nuestra investigación, ante todo es necesario señalar que este trabajo se inscribe en el marco del Proyecto de Investigación y Desarrollo titulado "La construcción de representaciones mediáticas de la Política Exterior Argentina a través de los medios de comunicación gráfica: el Observatorio de Política Exterior Argentina 2011-2017". En efecto, el proyecto se nutre de la labor del citado espacio que lleva más de trece años de actividad y producción de conocimiento ligado a la Política Exterior Argentina estando compuesto por estudiantes, graduados, docentes e investigadores.

El principal producto del Observatorio de Política Exterior Argentina (OPEA) son sus informes semanales sobre política exterior argentina. Estos últimos son documentos de seguimiento de las noticias de la política exterior argentina a través de cuatro fuentes: los boletines de prensa del Ministerio de Relaciones Exteriores y Culto de la República Argentina y los tres medios gráficos de mayor tirada nacional: Clarín, La Nación y Página 12. En efecto, la labor del observatorio implica la identificación, de forma semanal, de todas las noticias de política exterior argentina en las cuatro fuentes previamente referidas. A partir de dicha identificación se procede a redactar un nuevo producto que se divide en temas de agenda y relaciones bilaterales. Cabe subrayar que, los citados informes son un insumo central para la investigación que pretende impulsar el referido proyecto de investigación y, por ende, para el presente trabajo.

En lo que hace al diseño metodológico del mismo, esta contribución se vale tanto del análisis cuantitativo como cualitativo. Tal es así que, en pos de un primer acercamiento al tema y con vistas a precisar el objeto de estudio de nuestro trabajo, se procedió a desarrollar una tabla dinámica de Excel que recogiera todos los títulos de los informes semanales del OPEA de 2011 a 2015, de modo de identificar aquellos que se presentasen con mayor frecuencia por año y en todo el período.

Dichos títulos respondieron a la clasificación de relaciones bilaterales y temas de la agenda de política exterior argentina en los cuales se dividen los informes. Dicha tabla arrojó 
por resultado que los países con los que existió mayor asiduidad de noticias fueron Brasil, Estados Unidos, Uruguay y China. Mientras que los temas de agenda que se publicaron con mayor frecuencia fueron la Cuestión Malvinas, las Relaciones Económicas Internacionales y los Derechos Humanos.

En virtud de estos primeros hallazgos, el presente trabajo se aboca a analizar las relaciones político-diplomáticas argentino-brasileñas a la luz de sus representaciones mediáticas, prestando particular atención a cómo los medios gráficos argentinos han dado cuenta de las mismas en términos de cooperación o conflicto en el período 2011-2015, correspondiéndose dicho recorte temporal con el segundo gobierno de Cristina Fernández.

Al respecto, es importante mencionar que la construcción de tablas también permitió rastrear una serie de palabras clave que emergieron de los citados informes semanales en el contexto de la relación bilateral. Estas sacaron a la luz instancias de cooperación y conflicto entre ambos países. Con lo cual, la labor de índole cuantitativa originó el diseño de este trabajo a partir del estudio de frecuencias de los temas y relaciones bilaterales con mayor aparición en los informes del OPEA, así como de las variables y subvariables explicitadas. Mientras que, luego, se avanzó hacia una dinámica cualitativa para el análisis de las fuentes primarias y secundarias.

Entre las primeras se encuentran documentos oficiales de ambos países incluyendo comunicados de prensa, declaraciones conjuntas y acuerdos suscritos, entre otras. En cuanto a las fuentes secundarias, se consultó libros y publicaciones periódicas con referato nacionales e internacionales ligadas al objeto de estudio y, por supuesto, los informes del OPEA. En lo que respecta a las técnicas de recolección de información se optó por la observación de datos o documental. Mientras que las técnicas de análisis de datos que se emplearon son el análisis documental y el análisis de contenidos. Asimismo, se realizó una triangulación entre la información que brindan los informes del OPEA y aquella proveniente de las fuentes secundarias, especialmente de los analistas de la relación bilateral. Dicha triangulación permitió constatar algunas diferencias entre las representaciones mediáticas y la mirada analítica, así como profundizar algunos aspectos que en los informes sólo se relevan de modo general por su naturaleza ligada a los medios, y por ende, preponderantemente informativa.

En cuanto a la organización del trabajo, el mismo cuenta con cinco apartados, a los que se adiciona esta introducción y una conclusión. Un primer apartado aborda la evolución que tuvo la variable Diálogo Político Bilateral tras la llegada al poder de los gobiernos de Rousseff y Fernández, en líneas generales. En tanto, los apartados subsiguientes profundizan en distintas facetas de dicho diálogo: integración regional, agenda multilateral conjunta, derechos humanos y temas de seguridad y defensa. Vale destacar que, en cada uno de los mismos, se procura desentrañar el carácter cooperativo o conflictivo de la vinculación.

Para cerrar este punto, a modo de hipótesis afirmamos que, las representaciones mediáticas de la política exterior argentina, manifiestas en los informes del OPEA, dieron cuenta de una relación cooperativa entre Argentina y Brasil en lo que respecta a la variable Diálogo Político Bilateral. Al desmenuzar dicha variable se connota que la única subvariable que se ha comportado de modo mixto, es decir, cooperativo y conflictivo, dependiendo del caso, ha sido integración regional. Las otras tres subvariables estudiadas, es decir, agenda multilateral conjunta, derechos humanos y temas de seguridad y defensa, se manifestaron en clave cooperativa en el período bajo estudio. 


\section{El diálogo político bilateral entre Argentina y Brasil a la luz de los medios gráficos y los analistas}

Tras las elecciones presidenciales del 28 de octubre de 2007, Cristina Fernández sucedió a Néstor Kirchner, como presidente de los argentinos. La ex senadora se consagró como ganadora de la contienda electoral en la primera vuelta, con el 45,9\% de los votos, recabando un caudal de votos sobre veinte puntos porcentuales más que el ex presidente, tornándose manifiesto el apoyo del pueblo a la gestión del FpV. De allí que cuando Fernández asumió su cargo, lo hiciese con la intención de dar continuidad al proyecto iniciado cuatro años antes.

Algunos de los lineamientos de política exterior del nuevo gobierno quedaron ya explicitados en el discurso de asunción presidencial, en el cual se hizo referencia a América Latina como "nuestra casa" y al MERCOSUR como "nuestro espacio". Tal como evidencia dicha alocución, Sudamérica ocuparía un lugar de privilegio dentro de la agenda de política exterior y, como veremos a continuación, Brasil detentaría un lugar preponderante dentro de la misma (Discurso de la presidenta Cristina Fernández frente a la Asamblea Legislativa, 10/12/2007) ${ }^{6}$.

Cristina Fernández ejerció la Presidencia de la Argentina por dos periodos consecutivos. Su primer mandato (2007-2011) coincidió con la Presidencia en Brasil de Ignacio Lula Da Silva (2003-2011). Conforme con Actis (2015), en aquellos años, las relaciones bilaterales adquirieron una fuerte intensidad. A lo que agrega que, en esta coyuntura de profundización de la parceria estratégica, la dinámica del vínculo bilateral tuvo como grandes pilares la ampliación de las relaciones económicas, fundamentalmente en materia de comercio, inversiones y financiamiento, a su vez -y de manera interrelacionada- el establecimiento de dinámicos lazos políticos estructurados y motorizados por una fuerte diplomacia presidencial.

En efecto, en dicho período, las coincidencias ideológicas y políticas favorecieron que ambos mandatarios estuviesen predispuestos al diálogo. Al respecto, Correa da silva (2016) destaca que esto es verificable a partir de la cantidad de encuentros concertados, sea en Buenos Aires o Brasilia, donde si bien no siempre se llegó a un acuerdo, existió la voluntad de minimizar las diferencias y potenciar la relación estratégica. Aún más, conforme con la autora, las cúpulas del MERCOSUR, de la UNASUR y otras arenas multilaterales sirvieron de espacio para el diálogo entre los dos países, denotando el esfuerzo conjunto.

En tanto, en lo que respecta al segundo mandato de Fernández (2011-2015), al cual nos abocamos en esta investigación, el mismo coincidió con el recambio presidencial y la elección como primera mandataria del país vecino de Dilma Rousseff (2011-2016). Rousseff resultó triunfante en los comicios con el $55 \%$ de los votos y, al igual que Fernández, se convirtió en la primera mujer en ejercer la presidencia de Brasil.

Ahora bien, en virtud del vínculo personal así como también de la convergencia política e ideológica entre ambas mandatarias, una de las temáticas que sobresale por su frecuencia de aparición en los medios gráficos en el periodo que se extiende entre 2011 y 2015 es la que denominamos Diálogo Político Bilateral. Cabe destacar que, como parte de la misma, consideramos los contactos, visitas y encuentros protagonizados por las primeras

\footnotetext{
${ }^{6}$ Discurso disponible en https://www.cfkargentina.com/asuncion-de-cristina-kirchner-10-de-diciembre-de2007/. Último acceso en Marzo de 2019.
} 
mandatarias, así como por otros agentes y funcionarios de alto nivel de ambos países. Asimismo, bajo dicha denominación se incluyen los resultados de tales encuentros, especialmente plasmados en la firma de acuerdos y declaraciones conjuntas.

Al respecto, el Diálogo Político Bilateral entre ambos países ha sido reflejado por los medios gráficos como un espacio donde primó casi exclusivamente la cooperación. Decimos esto ya que, a partir de la lectura de los informes del OPEA, que recogen los encuentros de distintos niveles - presidencial, ministerial, de cancilleres-, que tomaron lugar durante el periodo de referencia, se evidencia que sólo unos pocos de los mismos permiten traslucir algún roce entre las partes. Como correlato, de dicha herramienta se desprende un amplio nivel de coincidencias entre ambos gobiernos. Todavía más, la asiduidad y la cantidad de encuentros de distinto tipo refleja la relevancia que ambos países le otorgaron a la relación bilateral.

Si nos enfocamos en los encuentros que tuvieron lugar a nivel presidencial, algunos como parte de visitas oficiales, otros que se dieron al margen de reuniones organizadas por distintos esquemas de cooperación regionales u organismos internacionales, entendemos que el encuentro entre Fernández y Rousseff que tomó lugar entre finales de enero y principios de febrero de 2011, fue uno de los de mayor relevancia. Decimos esto, en primer lugar, considerando que Rousseff arribó a Argentina en lo que fue su primera visita al exterior desde que había asumido la Presidencia de Brasil, en un gesto político de alto impacto que expone la importancia que dicho país otorga a su relación con la Argentina. En esta misma dirección, también hacemos hincapié en la relevancia de este encuentro en virtud de la cantidad y la variedad de temas abordados a lo largo del mismo, de la sintonía que mostraron las partes, de los actores que participaron del mismo -atendiendo a que Rousseff incluso se hizo espacio para entrevistarse con organizaciones de derechos humanos-, y por último, a sus resultados. En torno a estos últimos, durante la visita de la primera mandataria brasileña se firmaron catorce acuerdos de cooperación e integración en las más diversas áreas: tecnología, energía, igualdad de género, aprovechamiento de recursos hídricos y promoción del comercio, entre otras ${ }^{7}$. Asimismo, la visita también se convirtió en el escenario

\footnotetext{
${ }^{7}$ Convenio interinstitucional entre la Caixa econômica Federal del Brasil y el Ministerio de Planificación Federal, Inversión Pública y Servicios de la República Argentina; Declaración para la promoción de la igualdad de género y la protección de los derechos de las mujeres; Declaración sobre los aprovechamientos de los recursos hídricos compartidos en el tramo limítrofe del río Uruguay y su afluente el río Pepirí Guazú; Acuerdo complementario al acuerdo básico de cooperación técnica entre el gobierno de la República Federativa del Brasil y el gobierno de la República Argentina para la implementación del proyecto "fortalecimiento de las farmacopeas de Brasil y argentina y sus laboratorios nacionales de control para la creación de una farmacopea regional"; Protocolo adicional al acuerdo para la creación de la Comisión de Cooperación y Desarrollo fronterizo (CODEFRO) entre el gobierno de la República Federativa del Brasil y el gobierno de la República Argentina; Memorando de entendimiento entre el gobierno de la República Federativa del Brasil y el gobierno de la República Argentina para cooperación en planeamiento urbano y vivienda; Plan de acción conjunta entre el gobierno de la República Federativa del Brasil y el gobierno de la República Argentina para avanzar en la cooperación bilateral en el área de masificación del acceso a internet de banda ancha (2011 - 2015); Acuerdo entre el gobierno de la República Federativa del Brasil y el gobierno de la República Argentina para la construcción de un puente internacional sobre el rio Pepirí-guazú, entre las ciudades de Paraíso, Brasil, y San Pedro, Argentina; Memorándum de entendimiento para la promoción comercial conjunta entre el gobierno de la República Federativa del Brasil y el gobierno de la República Argentina; Memorando de entendimiento entre el gobierno de la República Federativa del Brasil y el gobierno de la República Argentina sobre la cooperación en el área de bioenergía, incluyendo los biocombustibles; Acuerdo de cooperación entre CNEA y CNEN sobre el proyecto de nuevo reactor de investigación multipropósito; Memorando de entendimiento entre el Ministerio de Minas y Energía de la República Federativa del Brasil y el Ministerio de Planificación
} 
propicio para la firma de una declaración conjunta en la que ambos gobiernos "Reafirmaron la importancia de la relación estratégica entre la Argentina y Brasil como eje constitutivo de la integración a nivel regional y reiteraron su compromiso con el proceso de integración bilateral como una política de estado en ambos países" (Declaración conjunta con ocasión de la visita oficial de la presidente Dilma Rousseff de la República Federativa de Brasil a la presidente Cristina Fernández de Kirchner a la República Argentina, Buenos Aires, 31/01/2011).

Es más, en julio de 2011, Fernández viajó a Brasil, devolviendo la visita de su par brasileña, y en aquella oportunidad se firmó un comunicado conjunto que reafirmó los términos de la declaración firmada en enero del mismo año. En el dicho documento, se consideró "la alianza estratégica como piedra fundamental para el éxito del proyecto común de integración" (Comunicado conjunto con ocasión de la visita oficial de la presidenta de la República Argentina Cristina Fernández de Kirchner a la República Federativa del Brasil, 29/07/2011).

Ahora bien, del relevamiento de noticias que se plasma en los informes del OPEA podemos concluir que, durante los encuentros al más alto nivel que tomaron lugar a lo largo del periodo bajo estudio, se buscó evitar los temas más álgidos y que suscitaran rispideces en el vínculo bilateral, que fueron relegados para su tratamiento por funcionarios de menor nivel. En tanto, en aquellos casos que los mismos fueron abordados, se buscó minimizar las diferencias y resaltar los objetivos comunes. A modo de ejemplo, podemos dar cuenta de que en la reunión previamente referida, en el mes de julio de 2011, se discutieron los problemas suscitados en el plano comercial, si bien las partes procuraron mostrar sintonía y minimizar las posturas encontradas. Tal es así que, en dicha oportunidad, la mandataria brasileña efectuó declaraciones en las que señaló que las dificultades entre Argentina y Brasil "son problemas de poca monta" (Informe No 179 del OPEA).

Sumando a lo hasta aquí expuesto, Pereyra Doval (2014) señala que durante el periodo bajo análisis no tuvieron lugar grandes conflictos entre ambos países en las áreas política, estratégica y diplomática sino que los mismos se circunscribieron al plano económico-comercial. En tanto, Gomez Saraiva y Zimmer (2016) alegan que en el campo político, la aproximación brasileña a las posiciones argentinas tuvo un importante papel compensador de los desencuentros en materia económico-comercial sobre los cuales se da cuenta en el próximo apartado.

Por otra parte, del seguimiento de los informes del OPEA también se desprende que con el correr del tiempo, se redujo la asiduidad de las visitas oficiales de las primeras mandatarias. De hecho, el mayor número de encuentros al más alto nivel entre 2014 y 2015 tuvo lugar en el marco de reuniones de diversas organizaciones internacionales y organismos regionales.

Por su parte, Actis (2015) establece que al comparar un conjunto de indicadores referentes al vínculo entre ambos actores en los periodos 2007-2010 y 2011-2014 queda en evidencia que la interacción bilateral atravesó, desde el año 2011, una etapa de baja intensidad relativa. Es decir un proceso de merma en la fortaleza y dinámica de los pilares (eco-

Federal, Inversión Pública y Servicios de la República Argentina sobre intercambio de energía eléctrica; Memorando de entendimiento entre el Ministerio de Ciencia y Tecnología de la República Federativa del Brasil y el Ministerio de Ciencia, Tecnología e Innovación Productiva de la República Argentina para cooperación en ciencia, tecnología e innovación en luz sincrotrón. 
nómicos y políticos) centrales en los cuales se había estructurado la relación desde principios del siglo XXI, ello en términos comparativos con lo acontecido en el período inmediatamente anterior.

Trayendo claridad sobre este punto, el autor menciona que un indicador importante a la hora de evidenciar la baja intensidad relativa de los lazos políticos se vincula con la propensión de los Jefes de Estados de visitar el país vecino, la cual se vio disminuida en términos comparativos con la etapa precedente. A lo que agrega, que algunos datos llamativos residen, por ejemplo, en que la primera mandataria argentina no asistió a la asunción de su par brasileña en el año 2011. Aún más, en línea con los resultados que arrojan los informes del OPEA, se destaca que Rousseff no realizó ninguna visita estrictamente bilateral en 2014 siendo la primera vez que sucedía desde que el Partido de los Trabajadores (PT) gobernaba Brasil.

Ahora bien, dejando de lado la comparación con el periodo inmediatamente precedente, debemos mencionar que dentro de la variable Diálogo Político Bilateral distinguimos distintos tópicos preponderantes o subvariables. En este sentido, es importante mencionar que se arribó a los mismos haciendo uso de la información que brindan los informes del OPEA la cual se volcó, una vez más, en una tabla dinámica. Esta última arrojó por resultado que los temas con mayor frecuencia de aparición en los medios gráficos, que se desprenden de la variable Diálogo Político Bilateral entre Argentina y Brasil, durante el periodo de referencia son: integración regional, agenda multilateral común, derechos humanos y los temas de defensa y seguridad.

\section{Integración regional: un tema central en términos del diálogo po- lítico bilateral}

Cabe destacar que dentro de la subvariable integración regional, los medios hacen mención con mayor frecuencia al espacio del MERCOSUR, donde tienen una mayor cantidad de apariciones las noticias que dan cuenta de la formación de un discurso común dentro del bloque, principalmente en torno a la negociación del acuerdo de relacionamiento y libre comercio con la Unión Europea. En torno al mismo, vale destacar que el mayor avance en el proceso de negociación se alcanzó en el año 2004 con un intercambio de ofertas. No obstante, desde entonces, las negociaciones se estancaron hasta 2010 cuando, tras la IV Cumbre América Latina, Caribe, Unión Europea (ALCUE), se impulsó un relanzamiento del proceso de negociación. A partir de entonces tuvieron lugar múltiples reuniones técnicas, si bien los avances fueron poco significativos en el período de estudio (Bartesaghi, 2015).

Profundizando en este punto, a finales de junio de 2010, representantes de ambos bloques se reunieron en Buenos Aires para trabajar sobre el acuerdo. Se esperaba que a partir de las reuniones del Comité de Negociaciones Bi-regionales (CNB) en 2011 se produjese un nuevo intercambio de ofertas; sin embargo, este nunca se concretó. A lo largo de 2012 se retornó a la mesa de negociación y, una vez más, existió la promesa de intercambiar ofertas. Luego, en 2013 se fijó un nuevo cronograma, señalando el último trimestre del año como el momento para avanzar con el demorado intercambio. Lo cierto es que los gobiernos de Fernández y Rousseff culminaron sin que el bloque pudiese arribar a una nueva presentación de ofertas. 
Los medios gráficos se han hecho eco de estos encuentros dando cuenta de las diversas reuniones bilaterales de distintos niveles en las que se intentó alcanzar una posición común entre Argentina y Brasil respecto a las negociaciones con la Unión Europea. Aún más, éstos han reflejado las reuniones concertadas entre funcionarios argentinos y brasileños para lograr consolidar una lista de desgravaciones arancelarias que luego serían presentadas ante el equipo negociador europeo. Si bien, como ya mencionamos, estas negociaciones no arrojaron resultados concretos.

Respecto a las razones del bloqueo negociador, Malamud (2012) lo asocia con que si bien todos los actores implicados manifestaron inicialmente la voluntad de cerrar rápidamente un acuerdo, cuando se negociaron los aspectos específicos, se tornó muy difícil arribar al mismo. Por su parte, Bartesaghi entiende que ambos bloques han logrado efectuar avances en el texto del capítulo político y también en aquel que versa sobre cooperación, mas no el capítulo de acceso a mercados, en particular en la oferta de comercio de bienes "que es donde se centra el principal escollo para avanzar en las negociaciones" (Bartesaghi, 2013: 12). Al respecto, señala que "para progresar en este campo, es necesario que las dos partes logren avances entre sus miembros, ya que ambos procesos de integración no han podido consensuar puertas adentro una nueva oferta para presentar a su contraparte" (Bartesaghi, 2013: 12). En el caso del MERCOSUR, el autor sostiene que esta situación es fruto de que Brasil y Argentina no consiguen ponerse de acuerdo sobre la presentación de una nueva oferta, debido a que en los últimos años se profundizaron las diferencias sobre la estrategia de inserción internacional más conveniente para el bloque.

Lo cierto es que las rispideces que este tema suscitó en el ámbito comercial incluso llegaron a trasladarse al terreno político-diplomático, tal como lo reflejan los informes del OPEA. Sin ir más lejos, durante una reunión en Caracas, en febrero de 2014, en la que se congregaron los miembros del MERCOSUR para negociar una propuesta común que luego se transmitiría a la Unión Europea, el secretario de Relaciones Económicas Internacionales argentino le exigió al gobierno brasileño una retractación pública por las declaraciones vertidas por parte de su embajadora, en una sesión del Parlamento Europeo. Esto en virtud de que, conforme con el funcionario argentino, la embajadora de Brasil ante la Unión Europea habría acusado a Buenos Aires de entorpecer la firma del tratado.

Conforme con los medios gráficos, la demanda presentada por quien encabezaba la delegación argentina causó malestar en la Cancillería brasileña la cual, por su parte, rehusó publicar una nota ministerial de desagravio, tal como exigía el funcionario argentino. De cualquier manera, para evitar que esta situación llegase a mayores, el gobierno brasileño aceptó la publicación de un comunicado a través de la embajada brasileña en Buenos Aires en el que se sostenía que "la repercusión de las declaraciones atribuidas a la representante de Brasil ante la UE, no corresponde con la evaluación positiva que Brasil hace del proceso de elaboración de una propuesta común del MERCOSUR, en el contexto de las negociaciones entre los dos bloques" (Informe No 291 del OPEA).

Con lo cual, podría evaluarse que este fue uno de los temas que motivó mayor distanciamiento y conflicto entre ambos países, lo que se enmarca en diferencias comerciales concretas y estrategias de negoicación que los informes del OPEA y otras fuentes secundarias relevan, si bien no son objeto de estudio de este trabajo.

Otro tema perteneciente a la agenda en materia de integración regional entre Argentina y Brasil que se ha visto reflejado en los medios gráficos es la integración en el marco de la UNASUR. Cabe recordar que el tratado constitutivo de la UNASUR se firmó en 2008 y que 
el artículo 2ㅇ del mismo establece que "La Unión de Naciones Suramericanas tiene como objetivo construir, de manera participativa y consensuada, un espacio de integración y unión en lo cultural, social, económico y político entre sus pueblos, otorgando prioridad al diálogo político, las políticas sociales, la educación, la energía, la infraestructura, el financiamiento y el medio ambiente, entre otros" (Tratado constitutivo de la Unión de Naciones Suramericanas, 2011).

UNASUR emergió como una iniciativa de fuerte impronta brasileña (Peña, 2009). En efecto, el rol que asumió Brasil como uno de los principales impulsores del proyecto debe entenderse como parte de su búsqueda de consolidar su proyección mundial mediante una intensa participación en los foros políticos y económicos regionales y multilaterales: una participación que apuntó por un lado a disminuir la vulnerabilidad del país, y por otro, a aumentar el propio poder (Bermudez Torres, 2011).

Los medios dan cuenta de que en el marco de la visita de Fernández a Rousseff en julio de 2011 ambas mandatarias celebraron la constitución de la UNASUR como factor de unidad, diálogo político y cooperación en América del Sur. De allí en más, podemos hacer referencia a la existencia de una multiplicidad de noticias que tratan sobre los diversos encuentros bilaterales en los que se ha abordado la situación del foro regional, las relaciones de los países bajo estudio con el bloque y la búsqueda de consensuar posiciones previo a una reunión de este foro de concertación o diálogo político (Bermúdez Torres, 2011). No obstante ello, vale aclarar que dichas noticias, recogidas en los informes del OPEA, en muchas oportunidades han carecido de especificidad en torno a los temas puntuales que fueron abordados en lo referente a la relación con el bloque. Por otra parte, la cantidad de apariciones de noticias sobre este esquema ha sido menor en términos comparativos con aqueIlas que hacen referencia al MERCOSUR.

Dejando de lado las noticias relativas a las dificultades que Argentina y Brasil han encontrado a la hora de consensuar una posición conjunta frente a la Unión Europea, y aquellas que dan cuenta de la integración en el marco de la UNASUR, otras noticias que pertenecen a la agenda regional, que en este caso se perciben como cooperativas son: la firma de acuerdos de asociaciones universitarias y el compromiso con la finalización del Plan Estratégico de Acción Social del Mercosur (PEAS).

Ahora bien, resulta paradójico que la incorporación de Venezuela al MERCOSUR presente la misma frecuencia de aparición en los informes del OPEA que las últimas dos noticias referidas, es decir, sólo una aparición, siendo que estas últimas revisten menor impacto o relevancia. Respecto al ingreso de Venezuela al bloque, debemos mencionar que distintos especialistas coinciden en que los factores que explican su incorporación han sido políticos. Ello en una instancia en la cual la República Bolivariana apostó por retirarse de la Comunidad Andina y profundizar su política contestataria hacia Estados Unidos. Mientras que, por su parte, el gobierno de Fernández precisaba obtener financiamiento internacional y Brasil apostaba por un mejor posicionamiento en el escenario regional (Bartesaghi, 2015).

Lo cierto es que, insistimos, la escasa cobertura respecto al ingreso de Venezuela al organismo regional llama la atención, considerando que la incorporación de este actor al bloque despertó un fuerte debate, fundamentalmente en lo referente a la legalidad de dicho proceso. Tal es así que hay quienes entienden que se trató de un ingreso forzado (Pastorino, 2017), o por la puerta trasera (Bernalette, 2012); esto a raíz de la crisis ocurrida en Paraguay que provocó la destitución del Presidente Fernando Lugo y de la posterior suspensión de ese país del MERCOSUR el 22 de junio de 2012. Cabe recordar que Paraguay se opuso al 
ingreso de Venezuela y que su suspensión como miembro del bloque permitió que el 29 de junio finalizara el proceso de ingreso del mencionado país en su ausencia (La Nación, 02/08/2012).

\section{El diálogo político bilateral en torno a la agenda multilateral con- junta}

En otro orden, respecto a la subvariable agenda multilateral conjunta entre Argentina y Brasil, ya en la referida visita de Rousseff a Fernández en enero de 2011, ambas mandatarias firmaron una declaración conjunta en la que subrayaron la importancia de democratizar los foros de gobernanza global, fortalecer el multilateralismo y avanzar en el proceso de reforma de las Naciones Unidas. En ese contexto, las mandatarias acordaron la relevancia de reformar el Consejo de Seguridad de las Naciones Unidas (Declaración conjunta con ocasión de la visita oficial de la presidente Dilma Rousseff de la República Federativa de Brasil a la presidente Cristina Fernández de Kirchner a la República Argentina, Buenos Aires, 31/01/2011) aunque son sabidas las diferencias de fondo de ambas posiciones a este respecto, en particular, en torno al eventual ingreso de Brasil con derecho a veto (Majdalani, 2013). Estas diferencias, que podrían pensarse como un factor de conflicto no se ven plasmadas por los medios gráficos en ese modo.

En tanto, en la cumbre presidencial de julio de 2011, tal como mencionan los informes del OPEA, se firmó un comunicado conjunto en el que se hizo referencia a la necesidad de democratizar los foros de gobernanza global y fortalecer el multilateralismo. Del mismo modo, se reconoció la necesidad de realizar esfuerzos conjuntos y coordinados para el éxito de la Conferencia de Naciones Unidas sobre el Desarrollo Sustentable (Rio + 20) que tuvo lugar en Río de Janeiro; se destacó la importancia del G20 y se enfatizó su apoyo al sistema multilateral de comercio y a la conclusión de la Ronda de Doha (Comunicado conjunto con ocasión de la visita oficial de la presidenta de la República Argentina Cristina Fernández de Kirchner a la República Federativa del Brasil, 29/07/2011). En torno a este punto, y en lo que respecta a la política exterior brasileña, Gomez Saraiva y Zimmer (2016) señalan que la defensa del multilateralismo apoyado en la idea de revisar las instituciones internacionales fue una constante del gobierno de Rousseff. Sin embargo, entienden que la búsqueda de un asiento en el Consejo de Seguridad de Naciones Unidas, que ocupó un lugar importante en el gobierno de Lula, perdió impulso durante el gobierno de su sucesora.

En la misma dirección, existen múltiples alocuciones que plasman que ambas mandatarias bregaron por una reforma de las instituciones financieras a nivel global y en las que se evidencian puntos de encuentro respecto a cómo debería haberse abordado la crisis financiera internacional. Sin ir más lejos, en el marco de la Cumbre del G20 que tomó lugar en México en 2012, Cristina Fernández y Dilma Rousseff establecieron una posición común al promover el crecimiento y el desarrollo como políticas para superar la crisis económica mundial, en contra de las recetas de ajuste impulsadas por actores europeos (La Voz, 18/06/12). Tal como señala Correa da Silva (2016:72), la presidenta argentina "imprimió un alto perfil en su accionar en los foros internacionales, cuando estos se convirtieron en un escenario importante para las denuncias respecto a la crisis mundial y una oportunidad de culpar a los países desarrollados por la difícil situación (...) Dichos discursos fueron acompañados por Dilma Rousseff quien se pronunció de manera un poco menos contundente". No 
obstante, una vez más, los informes analizados hacen alusión a la agenda multilateral común con poca frecuencia y sólo como un punto entre las diversas temáticas abordadas en las reuniones entre ambos países.

\section{Derechos humanos: una temática que atraviesa el diálogo político bilateral}

Como contrapartida a lo que ocurre con la agenda multilateral común, una temática que atraviesa el Diálogo Político Bilateral entre Argentina y Brasil, a la cual hacen referencia los medios gráficos, coincide con la subvariable Derechos Humanos. Sin ir más lejos, la prensa gráfica da cuentas de que ya en su primer encuentro, en enero de 2011, Fernández y Rousseff abordaron este tema; dejando plasmada su posición en la declaración conjunta que entonces firmaron. Esta establece: "el firme compromiso de sus gobiernos y pueblos con la promoción y protección de los derechos humanos" y "la convicción en la defensa de los principios de universalidad, indivisibilidad e interdependencia de los derechos humanos, tanto de los derechos civiles y políticos, como de los derechos económicos, sociales y culturales" (Declaración conjunta con ocasión de la visita oficial de la presidente Dilma Rousseff de la República Federativa de Brasil a la presidente Cristina Fernández de Kirchner a la República Argentina, Buenos Aires, 31/01/2011). Aún más, los medios gráficos -reflejados en los informes del OPEA- también dieron cuenta de que, como parte de su ya referida primer visita a la Argentina, Rousseff se entrevistó con las Madres de Plaza de Mayo y otras organizaciones de derechos humanos en la Casa Gobierno, lo que evidencia el interés que la primera mandataria brasileña le asignó desde un primer momento a este tema de la agenda bilateral.

En esta misma dirección, no podemos dejar de mencionar que ya en su discurso de toma de posesión, Rousseff manifestó su intención de esclarecer los cerca de 400 asesinatos y desapariciones de opositores al régimen militar, así como también de investigar a quienes hubiesen estados relacionados con las violaciones a los derechos humanos que tuvieron lugar en el país vecino durante la dictadura militar. Para comprender su interés en este tema, no debemos perder de vista que Rousseff fue una de las víctimas de la dictadura brasileña, en tanto integró una organización armada que enfrentó al régimen militar, lo que le valió ser torturada y permanecer presa por casi tres años. De allí su compromiso con este tema que, una vez más, se hace palpable con la creación de la Comisión de la Verdad, en 2012, que tendrá por fin investigar los crímenes cometidos durante dicha dolorosa etapa de la historia brasileña. Ahora bien, como podemos apreciar, la preocupación de la primera mandataria brasileña en torno a esta temática se encuentra en consonancia con lo que sucedía en la Argentina. Decimos esto ya que no sólo para las administraciones de Fernández sino para el conjunto de los gobiernos kirchneristas la problemática de los derechos humanos se convirtió uno de los principales ejes tanto de política interna como externa.

Dicho esto, otras noticias que forman parte de los informes del OPEA y que reflejan una relación cooperativa respecto a esta temática refieren a: la presentación de una propuesta conjunta en torno a la reforma del sistema interamericano, específicamente en materia de Derechos Humanos y la negociación, firma y posterior puesta en marcha del Memorándum de Entendimiento entre la República Argentina y la República Federativa de Bra- 
sil para el Intercambio de Documentación con vistas al Esclarecimiento de Graves Violaciones a los Derechos Humanos. Asimismo, se da cuenta de la creación de una Comisión Técnica sobre Derechos Humanos que engloba a los dos países y tiene por fin facilitar el intercambio de dicha documentación. Incluso se hace referencia a que la Argentina entregó a la Comisión Nacional de la Verdad de Brasil material que contienen información relativa al terrorismo de Estado practicado sobre ciudadanos brasileños desaparecidos en territorio nacional así como también sobre ciudadanos argentinos desparecidos en territorio brasileño durante las cruentas dictaduras que atravesaron ambos países.

\section{La agenda cooperativa en términos de seguridad y defensa}

En otro orden, dentro de la variable Diálogo Político Bilateral, encontramos otra subvariable que por su frecuencia de aparición en los informes del OPEA resulta importante. Hacemos referencia a los temas de defensa y seguridad, los cuales también han sido relevados como un área de cooperación entre ambos países. Aún más, otro dato que se desprende de los referidos informes reside en que el abordaje de estos temas se ha efectuado principalmente a nivel ministerial.

Desglosando los encuentros de alto nivel en los que se han abordado temas relativos a la defensa y la seguridad encontramos que, en la visita que el Ministro de Defensa de Brasil realizó a Buenos Aires, en febrero de 2011, este firmó junto a su par argentino un documento sobre cooperación en producción de vehículos y aeronaves militares en el que también se previó apoyo logístico en la campaña antártica. En tanto, la cooperación en materia de defensa también fue objeto de análisis durante la visita de Fernández a Rousseff, en julio de 2011. Conforme al comunicado conjunto que se emitió tras este encuentro, ambas partes "Reafirmaron la importancia de la relación estratégica en materia de Defensa entre la Argentina y el Brasil. Asimismo, celebraron la puesta en funcionamiento del Mecanismo de Diálogo Político Estratégico a nivel Viceministerial (MDPEVM), creado con el objeto de darle sustentabilidad y profundizar la cooperación en áreas de políticas de defensa entre Brasil y Argentina" (Comunicado conjunto con ocasión de la visita oficial de la presidenta de la República Argentina Cristina Fernández de Kirchner a la República Federativa del Brasil, 29/07/2011).

En el mes de septiembre de 2011, una nueva reunión de ministros de Defensa de ambos países brindó el marco propicio para que ambos funcionarios reafirmasen la "alianza estratégica entre ambos países". Entonces, en la que fue la primera visita oficial del ministro Amorim -a cargo de la cartera de Defensa- a la Argentina, se puso énfasis en la necesidad de: coordinar posturas y acciones en el ámbito del Consejo de Defensa Suramericano y otros foros multilaterales; profundizar los intercambios de formación y capacitación, especialmente en materia de ingeniería en defensa; así como también de incrementar la cooperación en materia de entrenamiento y apoyo logístico antártico. A su vez, estos funcionarios evaluaron la posibilidad de realizar ejercicios combinados con la eventual participación de terceros países, y la coordinación de acciones respecto de la Misión de las Naciones Unidas para la Estabilización de Haití (MINUSTAH). Fruto de dicho encuentro los ministros de Defensa suscribieron una declaración conjunta en la que reafirmaron la "alianza estratégica" entre los dos países. El documento también señala áreas de interés mutuo en materia de diálogo político-estratégico y cooperación política en defensa, así como en la cooperación 
en tecnología y producción para la defensa. Se destacan como puntos importantes de la declaración, la intención de mantener al Atlántico Sur como Zona de Paz y cooperación, libre de armas nucleares, y de incrementar la cooperación tecnológica y productiva. Como medida puntual en torno a esta última, se previó la futura producción en serie del vehículo Gaucho así como el posible desarrollo de vehículos blindados. En esta misma dirección, en septiembre de 2013, en un nuevo encuentro de las más altas autoridades de la cartera de defensa, éstas suscribieron una declaración de trabajo conjunto y anunciaron la "complementación en ciberdefensa" como un punto clave en la agenda bilateral.

Aún más, con referencia a los temas específicamente trabajados en los encuentros hasta aquí referidos, es menester hacer hincapié en el respaldo del gobierno brasileño al reclamo argentino sobre la cuestión Malvinas. Sumando en esta dirección, los informes dan cuenta de la decisión asumida por el gobierno de Brasil de no brindar colaboración a buques militares del Reino Unido, así como también de no prestar ayuda para la exploración de petróleo o energía en las islas.

Ahora bien, específicamente respecto a los temas de seguridad y defensa, llama la atención que en los distintos encuentros de alto nivel entre funcionarios de ambos países, sólo aparezca una mención velada al Consejo de Defensa Sudamericano. Respecto a este último, el mismo fue creado en el año 2008, a propuesta de Brasil, como órgano encargado de fomentar la consulta, la cooperación y la coordinación en materia de defensa en la UNASUR. Aún más, Gratius (2018: 3) entiende su conformación como un ejemplo del liderazgo brasileño que el autor define como "ambivalente" en tanto entiende "que oscila entre su proyecto global (o interés nacional) y su proyecto sudamericano (interés colectivo)". Lo cierto es que, la escasa cobertura de noticias en torno a la cooperación en materia de defensa, canalizada a través de este órgano regional, podría explicarse a la luz de lo que algunos especialistas comprenden como su ingreso "en compás de espera" durante este periodo (Gomez Saraiva y Zimmer, 2016). Puesto que, pese a los logros iniciales del Consejo, se perciben signos de estancamiento en torno al devenir de este órgano (Bragatti y Goncalvez, 2018).

\section{Conclusión}

Este trabajo propone analizar las relaciones político-diplomáticas argentino-brasileñas a la luz de sus representaciones mediáticas, prestando particular atención a cómo los medios gráficos argentinos han dado cuenta de las mismas en términos de cooperación o conflicto, en el período 2011-2015.

Con vistas a alcanzar tal objetivo, el principal insumo para poder efectuar nuestro análisis han sido los informes del OPEA, que incluyen las noticias de política exterior argentina publicadas en tres medios gráficos y los boletines de la Cancillería Argentina. Ahora bien, más allá de la relevancia que han detentado estos informes, como reflejo de las noticias de política exterior vertidas en los medio gráficos argentinos, a los fines de enriquecer el trabajo y poder presentar un estudio más acabado de los temas bajo análisis, hemos recurrido a la triangulación metodológica. Esto nos ha permitido contrastar los resultados que arrojan los medios gráficos con la visión de distintos especialistas. 
Partiendo de que el trabajo busca echar luz sobre las instancias de cooperación y conflicto en la relación político-diplomática bilateral, hemos analizado la variable Diálogo Político Bilateral a través de cuatro subvariables en función de su mayor aparición en los informes, y por ende, en la prensa escrita argentina.

Como exponen los informes del OPEA, las representaciones mediáticas en torno a las relaciones político-diplomáticas entre Argentina y Brasil resultan preponderantemente cooperativas en este período, dejando de lado la visión del otro como rival o contrincante. Tal como deviene de las noticias que dan cuenta de: los múltiples encuentros concertados, las declaraciones auspiciosas de los funcionarios gubernamentales, los acuerdos firmados y los comunicados conjuntos emitidos. Al respecto, es importante subrayar que el carácter cooperativo de la relación se sostiene independientemente del sesgo ideológico de los diferentes periódicos relevados para efectuar el análisis. Aún más, esta lectura es reafirmada por la literatura sobre el periodo donde se hace hincapié en la sintonía vigente entre las primeras mandatarias, la voluntad de profundizar la cooperación y minimizar las diferencias.

En términos más específicos, en lo que refiere a la agenda multilateral conjunta, el apoyo al multilateralismo ha sido una constante y un tema en el que ha existido un amplio grado de coordinación política entre ambos países. En esta instancia se destacan las noticias en las que se hace hincapié en la visión compartida en torno a la necesidad de reformar las instituciones internacionales y democratizar los organismos financieros. En este sentido, se subraya el carácter cooperativo de la agenda en torno a estos temas, más allá de los intereses particulares de cada una de las partes y de los temas en los que cada una de las mismas puso énfasis. Uno de los temas que podría haber supuesto un desencuentro como es el caso de la reforma del Consejo de Seguridad de Naciones Unidas, donde ambos países tienen posiciones encontradas, no resultó tal. Los analistas apuntan a que el tema tuvo menor relevancia en el gobierno de Dilma de Rousseff en comparación con su antecesor.

Por otra parte, en lo que respecta a la defensa de los derechos humanos, esta ha sido una cuestión de relevancia primaria para ambos gobiernos, convertida en uno de los grandes lineamientos tanto de política interna como externa de las administraciones de Fernández y Rousseff. Lo cual se comprende teniendo en consideración la historia personal de ambas mandatarias, las heridas que aún hoy permanecen abiertas en ambos países, muchos años tras la culminación de los gobiernos dictatoriales, pero también el ideario de los partidos que impulsaron su llegada al poder. En esta misma dirección, los más claros ejemplos de la cooperación en este tema resultan la firma de un Memorándum de Entendimiento para el Intercambio de Documentación con vistas al Esclarecimiento de Violaciones a los Derechos Humanos y la creación de una Comisión Técnica sobre Derechos Humanos que engloba a los dos países y tiene por fin facilitar el intercambio de dicha documentación.

En términos de seguridad y defensa, la agenda también resultó nutrida y ampliamente cooperativa. Lo cual se ha plasmado en las diversas reuniones que han tenido lugar entre funcionarios del más alto nivel, los acuerdos firmados y la relevancia de los temas abordados, siempre caros para la soberanía estatal. Incluso, se destaca la cooperación para hacer frente a las nuevas a amenazas a la seguridad como puede ser el caso de los ciberataques, o en otra área en la que Brasil posee amplia expertise, como lo es en materia de desarrollo de aeronaves. Ambos países, supieron acompañarse en aspectos claves de sus respectivas políticas exteriores, donde la Cuestión Malvinas y la militarización del Atlántico Sur fueron claves para Argentina. Asimismo, Argentina apoyó el liderazgo brasileño de MINUSTAH y el Consejo de Defensa Sudamericano, entre otros temas. 
Por último, las representaciones mediáticas en materia de integración regional se perciben como las únicas que presentan un balance mixto. Gran parte de la discusión en torno a esta temática giró en torno a la construcción de un discurso común dentro del MERCOSUR a la hora de negociar un acuerdo de libre comercio con la Unión Europea, un tema que ha suscitado rispideces entre las partes, no sólo en el campo económico-comercial sino, inclusive, en el terreno político-diplomático. Sin embargo, hablamos de un balance mixto dado que, a pesar de lo expuesto, las partes han manifestado su predisposición a profundizar la integración regional tanto en el marco del MERCOSUR como de la UNASUR, valorando positivamente el diálogo al interior de dicho foro.

En esta misma dirección, la sintonía entre las partes se ha evidenciado a la hora de apoyar la suspensión de Paraguay del bloque sudamericano, así como también de respaldar incorporación de Venezuela al mismo. Un tema que pese a la importancia política primaria que reviste, al hacer referencia a la inclusión de un nuevo socio en el organismo regional, con todas sus implicancias, obtuvo baja cobertura mediática, tal como reflejan los informes del OPEA.

\section{Bibliografía}

Actis, Esteban (2015), “La relación bilateral entre Argentina y Brasil (2011-2014). La confluencia de factores sistémicos y domésticos para una menor intensidad relativa en las interacciones", Estudos Internacionais, PUCMINAS, v. 3, № 1.

Bartesaghi, Ignacio (2013), "Las negociaciones comerciales entre el MERCOSUR y la Unión Europea: la visión desde un país pequeño", en Repensando la integración y las integraciones, Universidad Externado de Colombia: Colombia. Primera edición.

Bartesaghi, Ignacio (2015), "Algunas reflexiones sobre el MERCOSUR, Propuestas para una necesaria reformulación", Análisis \& Perspectivas, Konrad Adenauer Stifing, no 3.

Battistella, Darío (2015), "Théories des relations internationales 5e édition: 5e édition mise à jour", Presses de Sciences Po.

Bermudez Torres, Cesar (2011), "La integración regional a comienzos del siglo XXI: MERCOSUR y UNASUR", Revista Digital de Historia y Arqueología desde el Caribe, Universidad del norte, Barranquilla, v. 8, no 14

Bernal Meza (2008), "Argentina y Brasil en la Política Internacional: regionalismo y Mercosur (estrategias, cooperación y factores de tensión)", Revista Brasileira de Política Internacional, v. 51, № 2 .

Bernalette, Oscar (2012), "El tortuoso ingreso de Venezuela al Mercosur", International Center for trade and Sustainable Development.

Bizzozero, Lincoln (2003), "Los cambios de gobierno en Argentina y Brasil y la conformación de la agenda MERCOSUR", Nueva Sociedad, no 186, jul-agos.

Bragatti, Milton y Goncalvez, Rubem (2018), "Cooperación en el área de defensa en la UNASUR: un balance del Consejo de Defensa Sudamericano y sus límites", Revista Jurídica da Presidência Brasília, v. 20, no 120, Fev./Maio 
Comunicado conjunto con ocasión de la visita oficial de la presidenta de la República Argentina Cristina Fernández de Kirchner a la República Federativa del Brasil, Brasilia, 29 de julio de 2011. Disponible en: http://www.itamaraty.gov.br/es/notasa-la-prensa/14311-comunicado-conjunto-con-ocasion-de-la-visita-oficial-dela-presidenta-de-la-republica-argentina-cristina-fernandez-de-kirchner-a-larepublica-federativa-del-brasil-brasilia-29-de-julio-del-2011

Corrêa da Silva, Vera Lúcia (2016), Cambios y continuidades de la Política Exterior Argentina hacia Brasil durante los gobiernos de Néstor Kirchner y Cristina Fernández de Kirchner (2003-2015): el impacto del acercamiento de Argentina a Venezuela, Tesis Doctoral, Facultad de Ciencias Políticas y Relaciones Internacionales, Universidad Nacional de Rosario.

Creus, Nicolas (2014), "Una historia de idas y vueltas: los dilemas de la cooperación entre Argentina y Brasil", Gladys Lecchini (Comp.), La cooperación Sur-Sur en las políticas exteriores de Argentina y Brasil en el siglo XXI, Rosario: UNR Editora.

Declaración conjunta con ocasión de la visita oficial de la presidente Dilma Rousseff de la República Federativa de Brasil a la presidente Cristina Fernández de Kirchner a la República Argentina, Buenos Aires, 31 de enero de 2011. Disponible en: http://www.itamaraty.gov.br/es/notas-a-la-prensa/9558-declaracion-conjunta-por-ocasion-de-la-visita-de-la-presidenta-dilma-rousseff-a-la-republicaargentina-buenos-aires-31-de-enero-de-2011

Discurso de la presidente Cristina Fernández frente a la Asamblea Legislativa, 10 de diciembre de 2007. Disponible en: https://www.cfkargentina.com/asuncion-de-cristinakirchner-10-de-diciembre-de-2007/

Gomez Saraiva, Miriam y Tedesco, Laura (2001), “Argentina e Brasil: políticas exteriores comparadas depois da guerra fría", Revista Brasileira de política internacional, $\mathrm{v}$. 44, no 2, Brasília,, July/Dec.

Gomez Saraiva, Miriam y Zimmer Bom Gomez (2016), “Os limites da política externa de Dilma Rousseff para a America do Sul”, Relaciones Internacionales, n 50.

Gomis, L. (1991), Teoría del Periodismo, Barcelona: Paidós.

Gratius, Susanne (2008), “¿Hacia una OTAN Sudamericana? Brasil y un Consejo de Defensa Sudamericano", FRIDE, Comentario, abril

Herrera Vegas (2012), Jorge Hugo, “Convergencias y divergencias en las relaciones entre Argentina y Brasil", Revista Institucional, Bolsa de Comercio de Rosario, no 1516, Año $\mathrm{Cl}$, Rosario.

Horta, Julio (2013), “Representaciones mediáticas. Tres notas sobre los procesos semióticos en los medios masivos, Comunicaciones y medios", Instituto de Comunicación e Imagen, Universidad de Chile, $\mathrm{n}=28$.

Informe OPEA No 179. Disponible en: https://opeargentina.wordpress.com/

Ingram, Helen y Fiederlein, Suzzane (1988), "Traversing boundaries: a public policy approach to the analysis of foreign policy", The Western Political Quarterly, v. 41, n4.

Jaguaribe, Helio (1982), “Brasil-Argentina: Breve análisis de las relaciones de conflicto y 
cooperación", Estudios Internacionales, Año 15, nํ5, Santiago de Chile.

Keohane, Robert (1986), "Reciprocity in international relations", International organization, v. 40, no 1, p. 1-27.

Lasswell Harold D. (1931), "The Measurement of Public Opinion", American Political Science Review, no 25, 311-26

La Nación (02/08/2012), "Mercosur: ilegal ingreso de Venezuela”, Buenos Aires, Argentina. Disponible en: https://www.lanacion.com.ar/1495527-mercosur-ilegal-ingreso-de-venezuela.

La Voz (18/06/12), "La Presidenta ya participa de la primera sesión de la Cumbre del G-20" Córdoba, Argentina. Disponible en: https://www.lavoz.com.ar/politica/presidenta-ya-participa-de-primera-sesion-de-cumbre-del-g-20.

Majdalani, Carla (2013), "Peculiaridades de un multilateralismo austral. Argentina en el Consejo de Seguridad 2013-2014", Revista Nueva Sociedad, no 246, pp. 4-13.

Malamud, Carlos (2012), "UE y MERCOSUR: negociaciones sin futuro", Real Instituto Elcano, ,ARI No 101.

Milner, Helen (1992), International theories of cooperation among nations: strengths and weaknesses, World politics, v. 44, no 3, p. 466-496.

Miranda, Roberto (1988), “El análisis de la política exterior argentina desde la perspectiva de las relaciones internacionales", Cuadernos de Política Exterior Argentina, Centro de Estudios en Relaciones Internacionales de Rosario, Ed. CERIR, Rosario, 1988.

Miranda, Roberto (2001), "El cambio externo y las estrategias internacionales de la Argentina", Relaciones Internacionales, Universidad Nacional de la Plata, La Plata, no 21, 2001.

Pastorino, Ana (2017), “Algunas reflexiones sobre el ingreso de la República Bolivariana de Venezuela al MERCOSUR, su desempeño como socio pleno y su actual situación de ¿cesación? En sus derechos como tal", Consejo Uruguayo para las Relaciones Internacionales, Análisis no 5/17

Peixoto, Juliana y Loza, Jorgelina, "La relación Argentina Brasil: entre la administración de conflictos y las políticas de cooperación", Revista Estudios, No 18, Centro de Estudios Avanzados (CEA), Universidad Nacional de Córdoba.

Peña, Félix (2009), "La integración del espacio sudamericano. ¿La UNASUR y el MERCOSUR pueden complementarse?”, Revista Nueva Sociedad, no 219, Venezuela, enero-febrero.

Pereyra Doval, Gisela (2014), "Relaciones Argentina-Brasil: cooperación con algunas discordias”, Conjuntura Global, v. 3, n 2, 2014.

Perina, Rubén (1988), "El estado de la política exterior y las relaciones internacionales", en Perina, Rubén y Russell, Roberto (Comp.), Argentina en el mundo (1973-1987), GEL, Buenos Aires. 
Puig, Juan Carlos (Comp.) (1984), América Latina: Políticas Exteriores Comparadas, GEL, Buenos Aires.

Russell, Roberto, Tokatlian, Juan Gabriel (2002), "El lugar de Brasil en la política exterior argentina: la visión del otro", Revista Desarrollo Económico, v. 42, n 167, pp. 405-428.

Russell, Roberto; Tokatlian, Juan Gabriel (2005), "Mejor la cooperación que el poder", La Nación, 28 de febrero. Disponible en https://www.lanacion.com.ar/683355-mejor-la-cooperacion-que-el-poder.

Russell, Roberto; Tokatlian, Juan Gabriel (2013), “América Latina y su gran estrategia: entre la aquiescencia y la autonomía", Revista CIDOB d'Afers Internacionals, no 104, 2013.

Selcher, Wayne (1985), "Relaciones entre Argentina y Brasil en la década del 80Ñ de una cautelosa rivalidad a una competencia amistosa", Estudios Internacionales, Año 18, no 70, Santiago de Chile

Simonoff, Alejandro (2009), "Regularidades de la política exterior de Néstor Kirchner", CONfines de relaciones internacionales y ciencia política, v. 5, no 10, p. 71-86.

Tratado constitutivo de la Unión de Naciones Suramericanas, Quito, 11 de marzo de 2011

Williams, Robin (1947), "The reduction of intergroup tensions: a survey of research on problems of ethnic, racial, and religious group relations", Social Science Research Council Bulletin, 57, xi, 153. 